\title{
Prevalence of age-associated memory impairment and dementia in a rural community
}

\author{
F Coria, J A Gomez de Caso, L Minguez, F Rodriguez-Artalejo, L E Claveria
}

\begin{abstract}
To obtain accurate estimates of the prevalence of age-associated memory impairment, dementia, and Alzheimer's disease, a population study was carried out in Turégano, a rural community of 1011 inhabitants in the Segovia province of Spain. The study was divided into two phases: a door to door survey of the entire population aged 40 years and over (503 persons), followed by a clinical examination of suspected cases for positive and differential diagnosis of dementia and cognitive impairment. The prevalence of age-associated memory impairment was $3 \cdot 6 \%$ in individuals of 40 years and over and $7 \cdot 1 \%$ in individuals of 65 years and over, whereas dementia was found in $2 \cdot 6 \%$ and $5 \cdot 2 \%$, respectively. The prevalence rates of both clinical conditions increased with age. The most prevalent clinical category of dementia was dementia of Alzheimer type, which represented $1.8 \%$ and $3.8 \%$ of these two age groups. The corresponding figures for vascular dementia were $0.4 \%$ and $0.9 \%$ and for secondary dementia $0.4 \%$ and $0.5 \%$. Age-associated memory impairment is an age-dependent disorder with a high prevalence among the elderly; some of these patients may represent an early stage of Alzheimer's disease, suggesting that the prevalence of this disorder may be higher than previously estimated.
\end{abstract}

$(\mathcal{F}$ Neurol Neurosurg Psychiatry 1993;56:973-976)

Memory impairment and dementia are among the most frequent neurological disorders of the elderly; Alzheimer's disease accounts for most of these cases. ${ }^{1}$ As a consequence of the projected shift in the age distribution of the population in developed countries, it is expected that the prevalence of these disorders will increase in the future. ${ }^{1}$ This has renewed an interest in epidemiological studies to assess their socioeconomic consequences, and to identify environmental factors of aetiological relevance.

Most previous studies have focused on epidemiological aspects of dementia as an incapacitating chronic condition. ${ }^{1}$ While they may provide data to assess health care needs, they are unlikely to provide clues to environmental causal factors, since dementia is a pluriaetiological syndrome, and most dementing ill- nesses may remain oligosymptomatic for years before dementia develops. ${ }^{2-4}$

The Segovia Programme on Dementia is a multidisciplinary project for clinical and molecular research on dementing illnesses. In the initial phase, its main objective was to obtain accurate information on the prevalence of dementia. For this purpose, we have performed a door to door survey in Turégano, a rural community in the province of Segovia, Spain. This province has special geographic and demographic characteristics, such as a very stable population, an extensive health care network, and a single reference neurological clinic, which provides an ideal setting for epidemiological studies.

This is the first door to door survey performed in a defined community in Spain, using universally accepted standardised criteria for the diagnosis of dementia, thus allowing comparison of prevalence estimates with those from other countries. ${ }^{1}$ In addition, this is the first field study which specifically addresses the prevalence of age-associated memory impairment (AAMI), ${ }^{3}$ a clinical syndrome characterised by mild memory disturbances, which in some cases may represent a predementing stage of Alzheimer's disease..$^{2-4}$

\section{Methods}

The study was divided into two distinct phases: a field survey, and then a standardised clinical interview for positive and differential diagnosis of dementia.

The field study was a door to door survey of the entire population aged 40 years and over. Eligible persons were identified and localised from the municipal census, updated for this study in August 1990. The survey instrument was a Spanish version of the Hodkinson's test ${ }^{5}$; a 10 item cognitive examination with $100 \%$ sensitivity and $76 \%$ specificity for dementia (Rocca et al ${ }^{6}$ and our unpublished data). This examination was performed by trained university students, and took a mean of 5 minutes per person to be completed. The entire available population was surveyed throughout a 4 week period in August 1990.

The second phase was performed by a neurologist (FC) in hospital and included persons judged to be cognitively impaired on the basis of their performance on Hodkinson's test. We defined a score of 7 or less as indicative of cognitive impairment in our community. The clinical examination was performed 
with the aid of a standardised instrument (CEMED ${ }^{\circ}$ ), which is divided into three parts. The first assesses subjective complaints obtained from the patient and a close relative, and includes a semistructured general and neurological medical history, which contains in full the Hachinski's scale for vascular dementia, ${ }^{7}$ the Hamilton's scale for depression, ${ }^{8}$ a modified structured Spanish version of the informant-based dementia scale of Blessed et $a l,{ }^{9}$ and the global deterioration scale of Reisberg et al. ${ }^{10}$ The second part assesses the mental status with a set of cognitive performance tests, which includes a Spanish modified version of the mini mental state examination, ${ }^{11}$ complemented with nonequivalent items from the information memory concentration test. ${ }^{9}$ The third part includes a set of algorithms for positive and differential diagnosis of dementia using standardised criteria.

Positive diagnosis relied on the following definitions and criteria. Cognitive impairment is an objective deficiency of higher mental functions. Based on previous longitudinal studies, ${ }^{4}$ a person is considered cognitively impaired when he or she scores 4 or more on the dementia scale of Blessed et al and/or 26 or less on the Mini-Mental State Examination. Cognitive impairment may be connatal or acquired. In the latter case, it may also result from an organic brain disease or a psychiatric illness. AAMI is a clinical condition particularly frequent in the elderly which is characterised by complaints of forgetfulness and objective evidence of memory loss in neuropsychological tests, and has no identifiable cause with the presently available diagnostic methods. ${ }^{3}$ Dementia is a pluraetiologic neurological syndrome which fulfils the DSM-IIIR clinical criteria. Alzheimer's disease is a specific dementing illness, independent of the age at onset and the stage it has reached, whereas dementia of Alzheimer type (DAT) is an advanced stage in the course of Alzheimer's disease when the degree of cognitive impairment fulfils DSM-IIIR criteria for dementia.

Differential diagnosis between dementia and primary depression was made by inference from the data obtained by the CEMED, and complementary biochemical, electrophysiological, and radiological data when needed. Differential diagnosis between depression and organic cognitive impairment was aided by the Hamilton's scale and the DSM-IIIR criteria for depression and other affective disorders, whereas differential diagnosis between degenerative and vascular dementia was aided by the Hachinski's scale and MRI. The diagnosis of DAT was made by exclusion of other dementing disorders and the positive criteria established by the NINCDS-ADRDA group for probable Alzheimer's disease. ${ }^{13}$

The validity of the screening instrument to detect cognitive impairment and dementia was determined in a random sample of the target population (to be published). Statistical calculations were performed according to Fleiss. ${ }^{14}$ Confidence intervals were calculated assuming a Poisson distribution.

\section{Results}

On prevalence day (1 August 1990), Turégano had a total population of 1011 inhabitants, 503 were 40 years and over. The distribution of this group by age is shown in table 1 . Only five people (1\%) were living in a nursing home in or outside the province.

In the field survey 476 of 503 (94.6\%) were interviewed; 52 of these scored 7 or less on the Hodkinson's test and therefore entered the second phase. Of the target population $27(5.4 \%)$ were not available for interview, because of refusal to participate, immigration to other areas, or institutionalisation. To complete the survey, we contacted close relatives or medical institutions in and outside Segovia. The informants were requested by telephone to complete the dementia scale of Blessed et al. Information obtained in this way was sufficient to decide upon the mental status in 24 cases. Five of them were considered to be cognitively impaired and accepted for clinical examination. Thus information was lacking from only three $(0.6 \%)$ people.

A total of 57 persons $(11.3 \%$ of the target population) entered the second phase. True cognitive impairment was found in all except five cases (10.3\% of the target population). Apart from affective disorders, severe sensory deficiencies, and mental retardation, 38 $(66.6 \%)$ persons were found to have an acquired organic cognitive impairment. Of these, 25 were not demented (43.9\%) and 13 were demented $(22 \cdot 8 \%)$. In the non-demented group, there were patients with recognisable medical causes of brain dysfunction, but the great majority of them (18 of 25) had an amnestic syndrome of unclear aetiology, which is best categorised as AAMI. By the global deterioration scale, ${ }^{10}$ dementia was graded as severe (stages 6 and 7) in six cases and mild to moderate (stages 4 and 5) in the other seven cases.

A diagnosis could be established with confidence in all demented cases. The most frequent diagnostic category was DAT (nine cases); all of them displayed simple severe cortical atrophy on MRI. Multi-infarct dementia was found in two other cases; both of them had MRI evidence of multiple large and small size infarcts. Secondary dementia was also found in two cases; one of them, who had a severe, long lasting cobalamin deficiency, improved after appropriate treatment. The prevalence rates of these conditions by age and sex are shown in table 2 .

The prevalence rates of AAMI and dementia by age are shown in table 1 . AAMI was found in $3.6 \%(\mathrm{CI}=2 \cdot 1-5 \cdot 7)$ of the population of 40 and over and $7 \cdot 1 \%(\mathrm{CI}=$ $4 \cdot 0-11 \cdot 8$ ) in the population of 65 and over, whereas dementia was present in $2.6 \%$ (CI $=$ $1 \cdot 6-4 \cdot 4)$ and $5 \cdot 2 \%(\mathrm{CI}=2 \cdot 6-9 \cdot 3)$, respectively. The relative frequency of both conditions increase with age, and is higher in 
Table 1 Prevalence rates of $A A M I$ and dementia related to age and sex

\begin{tabular}{|c|c|c|c|c|c|c|c|c|c|c|c|c|}
\hline Age & $\begin{array}{l}\text { Popu } \\
N\end{array}$ & ion & $F$ & $\begin{array}{l}A A M I \\
N(\%)\end{array}$ & $M(\%)$ & $F(\%)$ & $\begin{array}{l}\text { Dementia } \\
N(\%)\end{array}$ & $M(\%)$ & $F(\%)$ & $\begin{array}{l}A A M I+ \\
N(\%)\end{array}$ & $\begin{array}{l}D A T \\
M(\%)\end{array}$ & $F(\%)$ \\
\hline $\begin{array}{l}40-54 \\
55-64 \\
65-74 \\
75-84 \\
85-94 \\
95+ \\
\geq 40 \\
\geq 65\end{array}$ & $\begin{array}{r}152 \\
141 \\
106 \\
78 \\
24 \\
2 \\
2 \\
503 \\
210\end{array}$ & $\begin{array}{r}76 \\
72 \\
51 \\
32 \\
10 \\
1 \\
242 \\
94\end{array}$ & $\begin{array}{r}76 \\
69 \\
55 \\
46 \\
14 \\
1 \\
261 \\
116\end{array}$ & $\begin{array}{l}- \\
3(2 \cdot 1) \\
3(2 \cdot 9) \\
7(9 \cdot 0) \\
4(16 \cdot 6) \\
1^{\star} \\
18(3 \cdot 6) \\
15(7 \cdot 1)\end{array}$ & $\begin{array}{l}\overline{1}(1 \cdot 4) \\
0 \\
3(9 \cdot 4) \\
1(10 \cdot 0) \\
0 \\
5(2 \cdot 0) \\
4(4 \cdot 3)\end{array}$ & $\begin{array}{l}- \\
2(2 \cdot 9) \\
3(1 \cdot 8) \\
4(8 \cdot 7) \\
3(21 \cdot 4) \\
1 \\
13(5 \cdot 0) \\
11(9 \cdot 5)\end{array}$ & $\begin{array}{l}- \\
2(1 \cdot 4) \\
3(2 \cdot 8) \\
5(6 \cdot 4) \\
3(12 \cdot 5) \\
\overline{13}(2 \cdot 6) \\
11(5 \cdot 2)\end{array}$ & $\begin{array}{l}- \\
0 \\
1(2 \cdot 0) \\
0 \\
2(20 \cdot 0) \\
\frac{-}{3(1 \cdot 2)} \\
3(3 \cdot 2)\end{array}$ & $\begin{array}{l}\overline{1}(1 \cdot 4) \\
2(3 \cdot 6) \\
5(10 \cdot 9) \\
1(7 \cdot 1) \\
\overline{9(3.4)} \\
8(6 \cdot 9)\end{array}$ & $\begin{array}{c}- \\
5(3 \cdot 6) \\
6(5 \cdot 7) \\
10(12 \cdot 8) \\
6(25 \cdot 0) \\
1^{\star} \\
28(5 \cdot 6) \\
23(10 \cdot 9)\end{array}$ & $\begin{array}{l}- \\
1(1 \cdot 4) \\
0 \\
3(9 \cdot 4) \\
2(20 \cdot 0) \\
0 \\
6(2 \cdot 4) \\
5(5 \cdot 3)\end{array}$ & $\begin{array}{c}- \\
3(4 \cdot 3) \\
5(9 \cdot 0) \\
8(17 \cdot 4) \\
4(28 \cdot 6) \\
1 \\
21(8 \cdot 0) \\
18(15 \cdot 5)\end{array}$ \\
\hline
\end{tabular}

$\star_{\text {Not computed because of small numbers }}$

$\mathbf{N}=$ total number of individuals; $M=$ males; $F=$ females; DAT = dementia of Alzheimer type; $M I D=$ multinfarct dementia; $\mathrm{SD}=$ secondary dementia.

women than in men. However, figures in the very elderly group may not be accurate because of the small number of subjects. The prevalence of severe and mild to moderate dementia in the group of 40 and over were $1.2 \%$ and $1.4 \%$, respectively. In the group of 65 and over, the corresponding figures were $2.9 \%$ and $3.3 \%$.

Information from relatives and examination of the hospital records disclosed that none of the demented patients and only six of the cognitively impaired non-demented cases had previously attended a neurological clinic.

\section{Discussion}

For this investigation, we introduced several methodological modifications with respect to other studies. (a) We have lowered the cut off age to 40 years, as Alzheimer's disease, the most prevalent dementing illness, is not always a disease of the elderly. ${ }^{1}$ (b) We distinguished between mild cognitive impairment and dementia. This distinction is of clinical and epidemiological relevance, since surveys based solely on the detection of dementia may underestimate the real incidence of most chronic dementing illnesses at their earliest stages, when dementia has not yet developed ${ }^{2}$ (c) We ruled out possible medical and psychiatric causes of mild cognitive impairment to isolate individuals with AAMI (d) We introduced $M R I$ in the differential diagnosis between multi-infarct dementia and DAT, as this technique has a higher sensitivity in detecting vascular lesions. ${ }^{15}$ (e) We made every effort to examine all persons forming the target population. In fact, this door to door survey succeeded in providing information on the prevalence of mild cognitive impairment, dementia, and type of dementing illness in $99.4 \%$ of individuals of a rural population in Spain. Therefore, the percentage of affected persons can be considered as an accurate estimate of the prevalence of these neurological syndromes in this setting.

Some patients with mild cognitive impair-

Table 2 Prevalence rates of clinical types of dementia by age and sex

\begin{tabular}{lllllll}
\hline & $40-64$ years & \multicolumn{3}{c}{65 and over } \\
& $N(\%)$ & $M(\%)$ & $F(\%)$ & $N(\%)$ & $M(\%)$ & $F(\%)$ \\
\hline DAT & $1(0.3)$ & 0 & $1(0.6)$ & $8(3.8)$ & $3(3.2)$ & $5(4.3)$ \\
MID & 0 & 0 & 0 & $2(0.9)$ & $1(1.0)$ & $1(0.9)$ \\
SD & $1(0.3)$ & $1(0.7)$ & 0 & $1(0.5)$ & $1(1.0)$ & 0 \\
\hline
\end{tabular}

Abbreviations are as in the text and Table 1. ment had identifiable medical or neurological causes of brain dysfunction; the remainder fulfil clinical criteria of AAMI. ${ }^{3}$ The total prevalence of AAMI in Turégano is $7 \cdot 1 \%$ of individuals of 65 years and over, stressing the social and medical importance of this syndrome among the elderly in this community. Regarding the nosological significance of this clinical syndrome, it has been shown that non-demented elders may show significant numbers of plaques at necropsy, ${ }^{2}$ morphologically and biochemically ${ }^{16}$ identical to neuritic plaques of Alzheimer disease suggesting that a significant number of aged people with memory impairment may represent an early stage of Alzheimer's disease. ${ }^{24}$ In fact, follow up studies in a different sample of 22 AAMI patients showed that nearly $40 \%$ developed DAT after a mean of two years from onset. ${ }^{4}$ These clinical and pathological data suggest that the prevalence of Alzheimer's disease (but not DAT) may be higher than previously estimated in this and other studies. If we sum up the number of AAMI and DAT patients found in our survey (table 1, last column), the prevalence rates for Alzheimer's disease would increase to $5.6 \%$ of the population aged 40 and over and to $10.9 \%$ of the population of 65 and over. These figures are more in accordance to those provided by studies designed to assess the prevalence of Alzheimer's disease independently of the degree of cognitive impairment. ${ }^{17} 18$ Extensive complementary examinations and outcome after prolonged follow up are now being evaluated in these patients to know which of them will actually develop dementia.

Prevalence rates of dementia in Turégano by age and sex are in the range of values found in other communities. Previous studies $^{119}$ show a wide range of values, from $0.9 \%^{19}$ to $6.2 \%{ }^{6}$ in the population below 65 , and from $1.9 \%^{20}$ to $16 \%^{21}$ in the population of 65 and over. If we take into account only those studies with similar design or diagnostic criteria, ${ }^{6}{ }^{19-25}$ variation of values decreases but are still wide, ranging from $2 \cdot 2 \%^{20}$ to $8 \cdot 4 \%{ }^{6}$ The question of whether these figures represent true regional differences in the prevalence of dementia or are attributable to methodological factors cannot be resolved at present due to differences in sample size, age distribution of the population, institutionalisation rates, case ascertainment sources, screening methods, and interpretation of diagnostic criteria, which greatly differ from one study to another, and may strongly bias prevalence estimates of dementia. ${ }^{1}$ 
Only a few studies have assessed the prevalence of specific types of dementing illnesses. ${ }^{1}$ Our results are in accordance with others in that DAT is the most prevalent dementing condition, and ranges from $2 \cdot 4 \%^{25}$ to $10 \cdot 3 \%^{18}$ of people aged 65 and over. Since the prevalence of DAT is strongly dependent on age, this wide variation may also be related to the different age distribution of the population, or sampling or screening methods of other surveys.

On the other hand, multi-infarct dementia represents a small fraction of demented cases with a prevalence rate less than $1 \%$ of persons of 65 and over. Eastern countries, like Russia, Japan, and China, have been reported to have an increased relative frequency of multiinfarct dementia compared with DAT. ${ }^{123}$ Nevertheless, the recent study by Zangh et $a l^{25}$ in Shanghai gave prevalence rates for both dementing conditions which are comparable to those found in Western countries.

Secondary dementia is as frequent as multi-infarct dementia in our survey. In other studies the relative frequency of this heterogeneous group is very variable, probably due to known variations in the geographic distribution of medical illnesses potentially causing dementia. ${ }^{1}$ In any case, secondary dementia is of particular medical interest since many diseases included in this group are potentially treatable. In fact, one of the two patients found in this study improved after appropriate treatment. This argues against nihilistic views of dementia and other cognitive complaints among the elderly, and demands prompt and complete neurological evaluation to determine treatable causes of dementia and cognitive impairment.

It is noteworthy that most demented people were not institutionalised, and none of them sought neurological assistance. This suggests that epidemiological studies exclusively based on the records of hospitals, nursing homes, and other health services may be strongly biased. Therefore, a door to door survey is a necessary step for accurate estimation of the prevalence of dementia, and even more so for AAMI and other mild cognitive disturbances.

We acknowledge the participation of the following individuals and institutions in this study: health and administrative authorities of Turégano for providing census data and faciliauthorities of Turegano for providing census data and facili-
ties for the field survey; INSALUD (Delegación Provincial de tegovia) for providing transport for patients; Horizonte Segovia) for providing transport for patients; Horizonte Cultural (University Student Association of Segovia), whose members participated in the field survey, the directorate of the
Segovia's General Hospital for providing facilities and nursing assistance, and the population of Turégano who enthusiastically participated in this study. Other participants in the Segovia Programme on Dementia are: A Berbel, I Corral, Duarte, C Gonzalez, MA Gonzalez, A Moreno, I Rubio, and F Uribe.
Supported by research grants from the Fondo de Supported by research grants from the Fondo de
Investigación Sanitaria $(89 / 1600)$ and Caja de Ahorros de Segovia (PIF Coria).

1 Jorm AF. The epidemiology of Alzheimer's disease and related disorders. London: Chapman and Hall. 1990.

2 Morris JC, McKeel, Jr DW, Storandt M. et al. Very mild Alzheimer's disease: informant-based clinical, psychometric, and pathologic distinction from normal aging. Neurology 1991;41:469-78.

3 Crook T, Bartus RT, Ferris SH, Whitehouse P, Cohen GD, Gershon S. Age-associated memory impairment: proposed diagnostic criteria and measures of clinical proposed diagnostic criteria and measures of clinical
change. Report of a National Institute of Mental Health work group. Dev Neuropsychol 1986;2:261-76.

4 Coria F, Gomez-Caso JA, Duarte J, et al. Age-associated memory impairment: Nosology and outcome. I Neurol 1992;239(Suppl 2):S66.

5 Hodkinson HM. Evaluation of a mental test score for assessment of mental impairment of the elderly. Age Ageing 1972;1:233-8.

6 Rocca WA, Bonaiuto S, Lippi A, et al. Prevalence of clinically diagnosed Alzheimer's disease and other dementing disorders: A door-to-door survey in Appignano, Macerata province, Italy. Neurology 1990;40:626-31.

7 Hachinski VC, Iliff LD, Zilhka E, et al. Cerebral blood flow in dementia. Arch Neurol 1975;32:632-7.

8 Hamilton M. A rating scale for depression. $f$ Neurol Neurosurg Psychiatry 1960;23:56-62.

9 Blessed G, Tomlison BL, Roth $M$. The association between quantitative measures of dementia and of senile change in the cerebral grey matter of elderly subjects. $\mathrm{Br}$ ¥ Psychiatry 1968;114:797-811.

10 Reisberg B, Ferris SH, DeLeon MJ, et al. The global deterioration scale for assessment of primary degenerative dementia. Am f Psychiat 1982;139:1236-9.

11 Folstein MF, Folstein SE, McHugh PR. "Mini-mental state" a practical method for grading the cognitive state of patients for the clinician. I Psychiat Res 1975;12: 189-98.

12 American Psychiatric Association. Diagnostic and statistical manual of mental disorders. 3rd ed. Revised. Washington, DC: American Psychiatric Association, 1987.

13 MacKahn G, Drachman D, Folstein M, Katzman R, Price D, Stadlan EM. Clinical diagnosis of Alzheimer's Price D, Stadlan EM. Clinical diagnosis of Alzheimer's under the auspices of department of Health and Human under the auspices of department of Health and Human Services Task For

14 Fleiss J. Statistical methods for rates and proportions. New York: John Wiley, 1981

15 Armstrong P, Keevil SF. Magnetic resonance imaging 2: clinical uses. $\mathrm{Br} \mathrm{Med} \mathcal{F}$ 1991;303:105-9.

16 Coria F, Castaño EM, Frangione B. Brain amyloid in normal aging, and cerebral amyloid angiopathy is antigenically related to Alzheimer's disease $\beta$-protein. Am $\mathcal{F}$ Pathol 1987;29:422-8.

17 Evans DA, Funkenstein HH, Albert MS, et al. Prevalence of Alzheimer's disease in a community population of older persons. $¥ A M A 1989 ; 262: 2551-6$.

18 Pfeffer RI, Afifi AA, Chance JM. Prevalence of Alzheimer's disease in a retirement community. $A m \mathcal{F}$ Alzheimer's disease in a retiremiol 1987;125:421-36.

19 Hofman A, Rocca WA, Brayne C, et al. The prevalence of dementia in Europe: A collaborative study of 1980-1990 findings. Int $\mathcal{f}$ Epidemiol 1991;20:736-48.

20 Schoenberg BS, Anderson DW, Haerer AF. Severe dementia. Prevalence and clinical features in a biracial US population. Arch Neurol 1985;42:740-3.

21 Heyman A, Fillembaum G, Proenitz B, Raiford K, Burchett B, Clark C. Estimated prevalence of dementia among elderly black and white community residents. Arch Neurol 1991;48:594-8.

22 Mölsä PK, Marttila RJ, Rinne UK. Epidemiology of dementia in a Finnish population. Acta Neurol Scand 1982;65:541-52.

23 Shibayama $\mathrm{H}$, Kasahara $\mathrm{Y}$, Kobayashi $\mathrm{H}$. Prevalence of dementia in a Japanese elderly population. Acta Psychiat dementia in a Japanese

24 Sulkava R, Wikström J, Aromaa A, et al. Prevalence of severe dementia in Finland. Neurology 1985;35:1025-9.

25 Zhang M, Katzman R, Salmon D, et al. The prevalence of dementia and Alzheimer's disease in Shanghai, China: Impact of age, gender, and education. Ann Neurol 1990; 27:428-37. 